\title{
Review Article \\ Small but Heavy Role: MicroRNAs in Hepatocellular Carcinoma Progression
}

\author{
Erbao Chen, Xiaojing Xu, Ruiqi Liu, and Tianshu Liu \\ Department of Medical Oncology, Zhongshan Hospital, Fudan University, Shanghai, China \\ Correspondence should be addressed to Tianshu Liu; liu_tianshu99@163.com
}

Received 8 December 2017; Accepted 8 February 2018; Published 22 May 2018

Academic Editor: Xin-yuan Guan

Copyright (C) 2018 Erbao Chen et al. This is an open access article distributed under the Creative Commons Attribution License, which permits unrestricted use, distribution, and reproduction in any medium, provided the original work is properly cited.

\begin{abstract}
Hepatocellular carcinoma (HCC), which accounts for $85-90 \%$ of primary liver cancer, is the fifth most common malignant tumor and the third leading cause of cancer-related deaths worldwide, but the pathological mechanism of HCC is still not fully elucidated. miRNAs are evolutionarily endogenous small noncoding RNAs that negatively regulate gene expression via posttranscriptional inhibition or target mRNA degradation in several diseases, especially human cancer. Therefore, discovering the roles of miRNAs is appealing to scientific researchers. Emerging evidence has shown that the aberrant expressions of numerous miRNAs are involved in many HCC biological processes. In hepatocarcinogenesis, miRNAs with dysregulated expression can exert their function as oncogenes or tumor suppressors depending on their cellular target during the cell cycle, and in tumor development, differentiation, apoptosis, angiogenesis, metastasis, and progression of the tumor microenvironment. In this review, we summarize current findings on miRNAs and assess their functions to explore the molecular mechanisms of tumor progression in HCC.
\end{abstract}

\section{Introduction}

As a major type of primary liver cancer (85-90\%), hepatocellular carcinoma (HCC) is one of the most notoriously invasive and aggressive human malignancies around the world and causes 500,000-600,000 deaths every year. Based on the high fatality of liver cancer (overall mortality-to-incidence ratio, 0.95), this type of epithelial cancer is still a major global health burden [1]. Despite the advancements in the diagnosis and therapy of HCC, the prognosis remains unsatisfactory due to high rates of relapse and metastasis. Etiologically, the development of HCC is prominently associated with fibrosis and cirrhosis, which is mainly caused by chronic hepatitis B virus (HBV) and/or hepatitis $\mathrm{C}$ virus (HCV) infection [2]. Other pathogenic reasons are diabetes $[3,4]$, obesity [5], intake of aflatoxin B1 [6], alcohol abuse [7], nonalcoholic fatty liver disease [8], and metabolic disease [9]. With exposure to these high risks, the liver suffers a series of hyperplastic and dysplastic disease stages and ultimately acquires a malignant phenotype with intrahepatic metastasis and extrinsic hepatic foci. Cancer progression is thought to involve the disordered regulation of oncogenes and tumor suppressor genes that have greatly influenced the advancement of knowledge on the molecular mechanisms of HCC development in certain processes such as the cell cycle, angiogenesis, apoptosis, and cell migration and metastasis. Therefore, the identification of different cellular factors and a clear understanding of the underlying molecular mechanisms that are involved in the development of tumor metastasis are critical.

As previously described, miRNAs can significantly contribute to the criteria. Substantial functional studies reveal molecular cellular phenomena linked to miRNAs as well as apoptosis, inflammation, cell cycle, and the tumor microenvironment in concert with various oncocytic molecular events facilitate tumor development, progression, and metastasis [10]. MiRNAs, a new class of short small (average 23 nucleotides in length) highly conserved noncoding RNAs, negatively control the expression of many target genes in HCC [11]. In addition, computational analyses show that miRNAs can regulate more than one-third of protein-coding gene expression transcriptionally and posttranscriptionally [12]. Thousands of miRNAs that bind to target mRNA through $3^{\prime}$-untranslated region (UTR) interactions have been identified. On the basis of data from a vast majority of miRNAs, aberrant oncogene activation and/or tumor suppressor inactivation commonly lead to HCC. Clarifying the complex 
characteristic of miRNAs could provide a clearer perception of HCC. In recent years, there has been a considerable advancement in the understanding of the role of miRNAs in cancer.

In this paper, we will summarize the role of miRNAs in hepatocarcinoma and in the progression of HCC, focusing on their regulatory roles as tumor suppressors and oncogenes in networks involving metastatic hepatocellular cancer.

\section{Brief Introduction of miRNA Biogenesis}

MiRNA genes are usually located in endonuclear noncoding regions, such as introns of coding genes. These genes are transcribed to shape hundreds of nucleotide long primary transcripts (pri-miRNAs) with RNA polymerase II, capped with 7-methylguanosine and polyadenylated. Subsequently, these pri-miRNAs are processed in the nucleus by the RNase III enzyme Drosha in assistance with its cofactor, the DiGeorge syndrome critical region gene 8 (DGCR8 also known as Pasha), into dozens of base-pair precursor miRNA (pre-miRNAs), which constitute a partly corresponding short hairpin structure [13]; then they are transported into the cytoplasm with assistance from the RAS-related nuclear protein through the RNA GTP-dependent transporter Exportin 5 (XPO-5). RNase III endoribonuclease Dicer and its partner $\mathrm{HIV}-1$ transactivate response RNA binding protein (TPBP) then guide pre-miRNAs into mature miRNAs composed of 20-24 nucleotides in the cytoplasm, which subsequently constitute the RNA-induced silencing complex (RISC) [14]. The miRNA/RISC complex then binds to the $3^{\prime}$-UTR of target mRNAs and regulates their expression depending on the level of base-pair matching [12]. RISC degrades target mRNA by perfect pairing with mRNA $3^{\prime}$-UTR. Otherwise, RISC inhibits target mRNA translation by imperfect pairing with mRNA $3^{\prime}$-UTR.

\section{Dysregulation of miRNAs in HCC}

MiRNAs are affirmed by pivotal regulatory functions and regulate many cellular biological processes; alterations in their expression contribute to neoplasm [84]. Many studies have revealed numerous miRNAs changes in HCC by multifarious microarray analysis, which can compare differential miRNA expression profiles between tumor and tumor-adjacent tissues, normal and cancer tissues, primary tissues and metastatic/recurrent foci, or miRNA expression differences induced by anticancer treatment. In addition, quantitative RT-PCR is often used in validating miRNA expression in clinical specimens. Many studies have reported several miRNAs related to hepatitis virus, differentiation, cell cycle, apoptosis, angiogenesis, invasion, and metastasis. These studies are summarized in Table 1.

\section{Aberrant miRNAs in the Cell Cycle}

Many studies show that dysregulation in the cell cycle is an important step in the development of hepatocarcinogenesis and miRNAs participate in this process. MiR-199a/b-3p can target tumor-promoting PAK4 to suppress HCC growth through inhibiting PAK4/Raf/MEK/ERK pathway [15]. MiR$302 \mathrm{~b}$ suppresses HCC hyperplasia due to associating with proliferation-related proteins, such as AKT2, CCND1, and CDK2, targeting the EGFR/AKT2/CCND1 pathway [16]. In addition, miR-302b suppresses HCC cell proliferation by targeting EGFR [17]. Ectopic expression of miR-184 restrains SOX7 protein, resulting in upregulation of c-Myc and Cyclin $\mathrm{D} 1$ expression and the phosphorylation of $\mathrm{Rb}$ to promote cell proliferation, tumorigenicity, and cell cycle progression [18]. MiR-200a suppresses the proliferation of HCC cells by induction of G1 phase arrest by CDK6 [19]. MiR-19a inhibits cell growth by targeting Cyclin D1 [20]. Notably, miR125a$5 p$ and miR-125b are upstream regulators of oncogenic sirtuin 7 (SIRT7), which is always overexpressed in HCC, and loss of SIRT7 induced p21WAF1/Cip suppresses cycle D1 expression, thus increasing the G1/S stage as well as suppressing proliferation [21]. The suppressor of variegation 39 homolog 1 (SUV39H1), the prototype of histone methyltransferase, is a major enzyme element for histone $\mathrm{H} 3$ lysine 9 trimethylation. The expression of miR-125b inhibits HCC development and metastasis by downregulating endogenous SUV39H1 expression at both messenger RNA and protein levels [22]. Interestingly, miR-140 inhibits hepatocellular genesis and arrests the cell G2/M phase by repressing TGFB receptor 1 (TGFBR1) and fibroblast growth factor 9 (FGF9) signaling through mitogen-activated protein kinase/extracellular signal-regulated kinase (MAPK/ERK) signaling [23]. Cancer cells can escape from the immune system to evade apoptosis and retain survival in the cancer microenvironment through many ways. CCNE1, CDC25A, CCND3, CDK4, and BTRC have been identified as direct targets of miR-497 and miR-195, which leads to aberrant cell proliferation in hepatocarcinogenesis [24]. The expression of miR-145 could induce G(2)-M cell cycle arrest and apoptosis through targeting multiple components of oncogenic insulin-like growth factor (IGF) signaling, including insulin receptor substrate- (IRS1-) 1, IRS2, and insulin-like growth factor 1 receptor signaling pathway [85]. MiR-451 inhibits the tumorigenicity of cells associated with the downregulation of cyclin D1 and c-Myc, through targeting direct suppression of IKK-beta [25]. MiR494 is overexpressed in human HCC tissues and joins in transformation by regulating the G1/S cell cycle transition through targeting mutation suppressors in colorectal cancer tumors [86]. The deregulation of miR-517a and miR-517c promotes HCC cell proliferation via targeting Pyk2 [26]. MiR371-5p facilitates the G1/S transition cell cycle by downregulating pre-mRNA processing factor 4 homolog B (PRPF4B) in human HCC cells [27]. MiR-221 overexpression is related to the status of tumor capsular infiltration in HCC clinical samples, and miR-221 inhibits cell growth and apoptosismediating G1/S-phase arrest [28]. MiR-221 also suppresses the p53 protein combining with MDM2 [29].

Overexpression of miR-1269 accelerates cell proliferation in HCC aiding in directly suppressing FOXO1 (MAPK/ERK) signaling [30]. MiR-148a expedites cell proliferation, cell cycle progression, cell migration, and anchorage independent growth by downregulating the PTEN protein [31]. MiR-1228 exerts pleiotropic function as an oncogene by promoting the cell cycle and cell mobility and negatively regulating 
TABLE 1: Processes involved in hepatocarcinogenesis and associated microRNAs.

\begin{tabular}{lcl}
\hline Processes & target & microRNAs \\
\hline & Cyclin D & miR-199a/b-3p, miR-19a, miR-451, miR-138, miR-302b \\
& p53 & miR-1228, miR-221, miR-1228 \\
Cell cycle and apoptosis & SOX7 & miR-148a, miR-93, miR-216a/217, miR-21 \\
& c-myc & miR-184, miR-24 \\
& AKT & miR-451, miR-148a-5p, miR-363-3p \\
& Bcl-2 & miR-133a, miR-105, miR-331-3p \\
Invasion and metastasis & SMAD & miR-125b, miR-224, miR-200 \\
& Beta-catenin & miR-125b, miR-224 \\
& MMP-2 & miR-200a \\
PIK3 & miR-29b \\
angiogenesis & VEGF & miR-126-3p, miR-7 \\
& VEGFA & miR-195, miR-146a \\
& AEG-1 & miR-302c, miR-26a \\
\hline
\end{tabular}

PTEN, phosphatase and tensin homolog; SOX7, SRY-related HMG-box; MMP-2, matrix metalloproteases 2; VEGF, vascular endothelial growth factor; and AEG-1, astrocyte elevated gene-1.

the expression of $\mathrm{p} 53$. Downregulation of $\mathrm{p} 53$ expression conversely increases expression of miR-1228, thereby acting as a positive feedback loop that contributes to hepatogenesis in HCC [32].

MiR-138 can regulate cyclin D3 and function as an oncogene in HCC [33]. Repression of hepatocyte nuclear factor3beta (HNF-3beta) by miR-141 suppresses hepatocellular cell proliferation and invasion and promotes apoptosis [34]. By degradation of the sex-determining region Y- (SRY-) box 7 (SOX7), overexpression of miR-24 promotes the proliferation and invasion of neoplasm cells [35]. Overexpression of miR-133a attributes to suppression of HCC cell growth by inhibition of Akt activation and the ERK signal pathway [36].

\section{Aberrant miRNAs in Apoptosis}

MiR-129-5p could inhibit the degradation of IkappaB-alpha, increase apoptosis, and reduce the migration of HCC cells by suppressing the valosin-containing protein (VCP) [37]. Overexpression of miR-122 diminishes the capacity of Galpha12 to increase the c-Met pathway to activate ERK, STAT3, and Akt/mTOR, suppressing cell proliferation by augmenting apoptosis [38, 39]. Similarly, through the c-Met/PI3K/Akt pathway, miR-93 promotes cell proliferation, migration, and invasion and also allows liver cancer cell to avoid apoptosis by directly inhibiting PTEN and CDKN1A expression [40]. In the same way, miR-105 suppresses cell proliferation by downregulating insulin receptor substrate-1, 3-phosphoinositidedependent protein kinase-1, and AKT1 directly [41]. Estrogen activates miR-23a and $\mathrm{p} 53$ expression via estrogen receptoralpha transcriptionally to control apoptosis in liver neoplasms [87]. Both let-7g and let-7i, mediated by the Bcl$\mathrm{xL}$ protein, deliver a concurrent effect to curb hepatoma cell proliferation and apoptosis [88]. MiR-125b promotes apoptosis by suppressing the antiapoptotic molecules Mcl1, Bcl-w, and IL-6R [42]. MiR-34a decreases mRNA protein stability to increase the expression of beta-catenin through degradation of long intergenic noncoding RNA UFC1 [43]. Forced overexpression of miR-144 remarkably reduces cell proliferation, increases apoptosis, and suppresses migration and invasion of HCC cells [89]. A proliferation-inducing ligand (APRIL), a member of the tumor necrosis factor (TNF) superfamily, is a newly discovered target of miR-383, contributing to tumor apoptosis [44]. MiR-34a suppresses cell growth, migration, and invasion; meanwhile, it increases cellular apoptosis and caspase activity in HCC cells [50]. Cells with high expression of miR-106a have stronger invasive ability, quicker cell cycle progression, and less apoptosis compared with the low expression cell line [90]. MiR-221 exerts inhibitory effect on apoptosis by targeting Bmf. Patients who have miR-221 overexpression are associated with a more lethal pathological pattern and less time to recurrence after surgery [45]. The data described above are summarized in Table 2 .

\section{Aberrant miRNAs during Invasion and Metastasis}

Invasion and metastasis, two important features of tumors, are the most lethal reasons for neoplasm relapse and poor prognosis [10]. Some patients stem from a background of chronic hepatic diseases like fibrosis. One study found that miR-125b functions with an inhibitory effect on epithelialmesenchymal transition (EMT) and EMT-associated signaling pathway by targeting small mothers against decapentaplegic (SMAD) 2 and 4 [91]. MiR-181a has a critical role in inducing hepatocyte EMT, which is a good substitute for the TGF- $\beta$-induced effect. VASH 2 expression mediated by miR200 in HCC cells expedites the malignant transformation of liver tumors by inducing EMT [46]. MiR-200a functions as an important regulatory inhibition factor in EMT by targeting the beta-catenin pathway [47]. CDC42, CDH1, PAK2, and BCL-2 are validated as targets of miR-224, which exhibits increased expression of both mRNA and protein 
TABLE 2: Aberrant miRNAs associated with the cell cycle and apoptosis.

\begin{tabular}{|c|c|c|c|}
\hline MicroRNAs & Type of deregulation & Target & Reference \\
\hline miR-199a/b-3p & Downregulated & Cyclin D1 & [15] \\
\hline miR-302b & Downregulated & EGFR, Cyclin A, Cyclin D1, CDK2 & {$[16,17]$} \\
\hline miR-184 & Upregulated & SOX7 & {$[18]$} \\
\hline miR-200a & Downregulated & CDK6 & {$[19]$} \\
\hline miR-19a & Downregulated & Cyclin D1 & {$[20]$} \\
\hline $\operatorname{miR} 125 a-5 p, \operatorname{miR}-125 b$ & Downregulated & sirtuin7, SUV39H1 & {$[21,22]$} \\
\hline miR-140 & Downregulated & TGFBR1, FGF9 & {$[23]$} \\
\hline miR-497, miR-195 & Upregulated & CCNE1, CDC25A, CCND3, CDK4, & {$[24]$} \\
\hline $\operatorname{miR}-451$ & Downregulated & cyclin D1, c-Myc & {$[25]$} \\
\hline miR-517a, miR-17c & Upregulated & Pyk2 & {$[26]$} \\
\hline miR-371-5p & Downregulated & PRPF4B & {$[27]$} \\
\hline miR-221, miR-1228 & Upregulated & p53 & {$[28,29]$} \\
\hline miR-1269 & Upregulated & FOXO1 & {$[30]$} \\
\hline miR-148a & Upregulated & PTEN & {$[31]$} \\
\hline miR-1228 & Upregulated & p53 & {$[32]$} \\
\hline miR-138 & Upregulated & Cyclin D3 & {$[33]$} \\
\hline miR-141 & Downregulated & HNF-3beta & {$[34]$} \\
\hline miR-24 & Upregulated & SOX7 & {$[35]$} \\
\hline miR-133a & Upregulated & AKT, ERK & {$[36]$} \\
\hline miR-129-5p & Downregulated & VCP & [37] \\
\hline miR-122 & Upregulated & Galpha12 & {$[38,39]$} \\
\hline miR-93 & Upregulated & PTEN, CDKN1A & {$[40]$} \\
\hline miR-105 & Upregulated & AKT1 & {$[41]$} \\
\hline miR-125b & Upregulated & Bcl-w, IL6R & {$[42]$} \\
\hline miR-34a & Upregulated & UFC1 & {$[43]$} \\
\hline miR-383 & Downregulated & APRIL & {$[44]$} \\
\hline miR-221 & Downregulated & $\mathrm{Bmf}$ & {$[45]$} \\
\hline
\end{tabular}

levels in HCC cells as well as in HCC tissues [48]. MiR-122, which comprises around $70 \%$ of the liver's total miRNAs, promotes hepatic fibrogenesis by targeting Kruppel like factor (KLF6); moreover, restoration of miR122a reduces the cancer incidence in mice through inhibiting EMT [49]. MiR-451 inhibited cell growth, induced G0/G1 arrest, and promoted apoptosis. Importantly, miR-451 can inhibit EMT [92].

MiR-329 significantly regulates cell invasion by targeting bromodomain containing 4 (BRD4) but has no effect on cell proliferation and apoptosis [51]. MiR-362-5p works through CYLD to activate the NF-kappaB signaling pathway to increase cell proliferation, clonogenicity, migration, invasion, and metastasis, which are associated with HCC progression [52].

Vascular invasion provides a direct route for tumor metastasis. MiR-494 can trigger gene silencing of multiple invasion-suppressor miRNAs by inhibiting genomic DNA demethylation by direct targeting of TET1, thereby leading to tumor vascular invasion. Metastasis is a complex process that involves multiple steps. By downregulating the antitumor genes tensin homology protein (PTEN) and cyclindependent kinase inhibitor 1 (CDKN1A), miR-93 can promote cell proliferation, migration, and invasion by activating the c-Met/PI3K/Akt signaling pathway; additionally, antimiR-93 transfection makes HCC cells more sensitive to sorafenib and tivantinib treatment [40]. Phosphatase and tensin homolog (PTEM) also is validated as a functional target of miR-216a/217. In combination with PTEM and decapentaplegic homolog 7 (SMAP7), miR-216a/217 raises the stem-like cell population and promotes the metastatic ability of HCC cells through activating the PI3K/Akt and TGF- $\beta$ pathways $[53,54]$. MiR-221 modulates the tumor suppressor HDAC6 through coordinated JNK/c-Jun and nuclear factor kappa B (NF-kappaB) signaling during HCC development and progression [55]. MiR-657 directly binds to the transducin-like enhancer protein 1 (TLE1) $3^{\prime}$-UTR and contributes to hepatic carcinogenesis by activating NFkappaB pathways [56]. NF-kappaB is a critical factor linking inflammation and metastasis. MiR-195 exerts its suppressive function by decreasing the expression of multiple NF-kappaB downstream effectors by directly targeting IKK alpha and TAB3 [57]. MiR-331-3p promotes cancer cell proliferation and EMT-mediated metastasis through suppression of the $\mathrm{PH}$ domain and leucine-rich repeat protein phosphatasemediated dephosphorylation of Akt [58]. Some tumor suppressor miRNAs exert their functions in multiple ways. Restoration of miR-7 inhibits HCC cell growth, migration, and metastasis through regulating the phosphoinositide 3kinase (PI3K)/Akt pathway targeting phosphoinositide 3kinase catalytic subunit delta (PIK3CD), rapamycin (mTOR), 
TABLE 3: Aberrant miRNAs associated with invasion and metastasis.

\begin{tabular}{lccc}
\hline MicroRNAs & Type of deregulation & Target & Reference \\
\hline miR-200 & Upregulated & VASH2 CDC42, CDH1, PAK2, BCL-2 \\
miR-224 & Downregulated & CDC42, CDH1, PAK2, BCL-2 & KLF6 \\
miR-122 & Downregulated & Beta-catenin & [48] \\
miR-200a & Upregulated & BRD4 & [50] \\
miR-329 & Upregulated & CYLD & {$[51]$} \\
miR-362-5p & Upregulated & PTEN, CDKN1A \\
miR-93 & Upregulated & PTEM, SMAP7 & [52] \\
miR-216a/217 & Upregulated & HDAC6 & TLE1 \\
miR-221 & Upregulated & [53,54] \\
miR-657 & Upregulated & [55] \\
miR-195 & Downregulated & IKK alpha, TAB3 \\
miR-331-3p & Upregulated & AKT \\
miR-7 & Downregulated & PIK3CD \\
\hline
\end{tabular}

and p70S6K $[59,60]$. The data described above are summarized in Table 3.

\section{Aberrant miRNAs in Angiogenesis}

MiR-214 contributes to cancer cell apoptosis, cell cycle, and angiogenesis by mediating the hepatoma-derived growth factor (HDGF) [61]. Overexpression of miR-125b in HCC cells decreases placenta growth factor expression and regulates the angiogenesis index [62]. MiR-29b exerts its antiangiogenesis function by suppressing matrix metalloproteinase-2 (MMP2) expression [63]. Expression of human sulfatase-1 (hSulf-1) and PTEN mediated by miR-21 lead to activation of Akt/ERK pathways and EMT in HCC cells and finally enhance the activity of HCC cell proliferation and movement and promote HCC xenograft tumor growth [64]. Through activating the PIK3C2alpha/Akt/HIF-1alpha/VEGFA pathway, miR-26a acts in the degradation of vascular endothelial growth factor A (VEGFA) to modulate angiogenesis [65]. MiR-195 also prevents tumor angiogenesis and metastasis by interacting with vascular endothelial growth factor (VEGF) and the prometastatic factors VAV2 and CDC42 [66]. FGF2 and VEGFA are the two most potent angiogenic factors. MiR503 deters neoplasm angiogenesis via targeting FGF2 and VAGFA [67]. Upregulation of miR-146a strengthens the angiogenic activity of endothelial cells by stimulating plateletderived growth factor receptor-alpha (PDGFRA) [68]. MiR126-3p contributes to metastasis and angiogenesis in HCC due to degradation of LRP6 and PIK3R2 [69]. MiR-26a reduces tumor angiogenesis of HCC through hepatocyte growth factor-cMet (HGF-cMet) signaling [70]. MiR-302c suppresses tumor growth in HCC through inhibition of EMT [71]. By inhibiting vascular endothelial growth factor A (VEGFA) and astrocyte elevated gene-1 (AEG-1), miR497 suppresses microvessel densities in xenograft cancers [72]. Interestingly, there is a negative feedback loop between polycomb repressive complex (PRC2) and miR-101 that affects the progression and metastasis of HCC. MiR101 is repressed by PRC2 through $\mathrm{c}-\mathrm{Myc}$ signaling, although in contrast, the expressions of EZH2 and EED, the two subunits of PRC2, are inhibited by miR-101 [73]. In essence, these results suggest a balancing act between tumor suppressors and oncogenes. Another study shows miR-148a-5p functions by directly targeting and inhibiting Myc expression, whereas miR-363-3p destabilizes Myc protein by directly targeting and inhibiting USP28. However, inhibition of miR-148a$5 p$ or miR-363-3p induces hepatocarcinoma by promoting G1-S-phase cell cycle transition, whereas their activation has the opposite effect [74]. MiR-139 interacts with Rho-kinase 2 (ROCK2) and reduces its expression in HCC cells line. Downregulation of miR-139 improves the invasive capacity of HCC cells in vitro and HCC metastasis in vivo [75]. MiRNA188-5p suppresses cancer cell proliferation and metastasis in vitro and in vivo by interacting with fibroblast growth factor 5 (FGF5) [76].

\section{Role of miRNAs in the Microenvironment}

Exosome-mediated miRNA transfer is an important mechanism of intercellular communication in HCC cells [93]. MiR-375 suppresses hepatoma cell growth targeting astrocyte elevated gene-1 (AEG-1) [77]. MiR-122 affects the metabolism of carcinoma cells through pyruvate kinase M2 [78]. It demonstrates that STAT3 signaling is a core pathway mediating human immune suppression in the tumor microenvironment. Micro-146a, an important downregulator of immune activity, exerts a negative effect on tumor development in HCC cells by promoting the expression of STAT3 activationassociated cytokines, like TGF- $\beta$, IL-17, VEGF, and I IFN, leading to HCC-induced NK cell dysfunction [79]. Abnormal autophagy activity decreases miRNA-224 targeting of Smad4 to accumulate cell migration and tumor formation [80, 81]. Enhanced TGF- $\beta$ activity suppresses the expression of miR$34 \mathrm{a}$, leading to a high production of chemokine CCL22, which recruits regulatory $\mathrm{T}$ (Treg) cells to facilitate cancer cell immune escape [82]. Li et al. demonstrated that miR148a expression was significantly lower in a cancer stem celllike subtype, which is clinically aggressive and associated with poor survival, than in other subtypes. Moreover, the miR-148a-ACVR1-BMP-Wnt circuit could profile/determine 
TABLE 4: Role of miRNAs in the tumor microenvironment.

\begin{tabular}{|c|c|c|c|}
\hline MicroRNAs & Type of deregulation & Target & Reference \\
\hline miR-214 & Upregulated & HDGF & {$[61]$} \\
\hline miR-125b & Upregulated & placenta growth factor & {$[62]$} \\
\hline $\operatorname{miR}-29 b$ & Downregulated & MMP-2 & {$[63]$} \\
\hline miR-21 & Downregulated & hSulf-1, PTEN & {$[64]$} \\
\hline miR-26a & Downregulated & VEGFA & {$[65]$} \\
\hline miR-195 & Downregulated & VEGF, VAV2, CDC42 & {$[66]$} \\
\hline miR-503 & Downregulated & FGF2, VAGFA & {$[67]$} \\
\hline miR-146a & Upregulated & PDGFRA & [68] \\
\hline miR-126-3p & Upregulated & LRP6, PIK3R2 & [69] \\
\hline miR-26a & Downregulated & c-MET & {$[70]$} \\
\hline $\mathrm{miR}-302 \mathrm{c}$ & Downregulated & VEGFA, AEG-1 & {$[71]$} \\
\hline $\mathrm{miR}-497$ & Downregulated & PRC2 & {$[72]$} \\
\hline miR101 & Downregulated & EZH2, EED & {$[73]$} \\
\hline miR-148a-5p/miR-363-3p & Downregulated & MYC & {$[74]$} \\
\hline miR-139 & Downregulated & ROCK2 & {$[75]$} \\
\hline miR-188-5p & Downregulated & FGF5 & {$[76]$} \\
\hline miR-375 & Downregulated & AEG-1 & {$[77]$} \\
\hline $\operatorname{miR}-122$ & Upregulated & pyruvate kinase M2 & {$[78]$} \\
\hline miR-146a & Upregulated & TGF- $\beta$, IL-17, VEGF, I IFN & {$[79]$} \\
\hline $\operatorname{miR}-224$ & Upregulated & Smad4 & {$[80,81]$} \\
\hline $\operatorname{miR}-34 a$ & Downregulated & CCL22 & {$[82]$} \\
\hline miR-148a & Downregulated & ACVR1 & {$[83]$} \\
\hline
\end{tabular}

an advanced clinical stem cell-like subtype of HCC [83]. The data described above are summarized in Table 4.

\section{Conclusions}

Sustained dysregulation of miRNAs, some of which act as oncogenes or tumor suppressors, plays an unequivocal role in the development of HCC. Its role in HCC development and metastasis has been clearly reported and supported by evidence from various lines of studies [33]. MiRNAs function as a new tiny class of gene regulators at the translation and/or posttranscriptional level. They exhibit different expression patterns in various types of cancers [34]. The dysregulation of miRNAs has been demonstrated to play a significant effect on cell cycle, apoptosis, invasion, migration, and metastasis in HCC through the interaction of cellular signaling networks. In the past three years, lots of progress has been made in understanding the accurate mechanisms for aberrant miRNA profiles; meanwhile, more targets and upstream regulatory molecules of these dysregulated miRNAs have been reported. In this review, we summarize the role of miRNAs in carcinogenesis and progression of HCC and supply a more distinct comprehensive picture of their status in gene interaction signaling pathways. Collectively, the investigative studies have resulted in a better understanding of cancerrelated miRNA functions and their roles as tumor suppressors and oncogenes. Given the implication of a large number of miRNAs in the control of key tumor suppressors and oncogenes, the deregulation of specific miRNAs has been shown to greatly influence HCC development, invasiveness, prognosis, and treatment response. From a diagnostic point of view, high stability of miRNAs in circulation makes them perfect biomarkers.

However, many studies observe abnormally expressed miRNAs in HCC, associated with the identification of miRNA clinical signatures, which are correlated with HCC progression or metastasis; this makes them potential diagnostic and/or prognostic markers. One of the biggest challenges is that miRNA clinical signatures are always validated in small samples. Therefore, larger specimens should be used to test precisely the hundreds of miRNAs in future studies. Nevertheless, using these miRNAs to achieve clinical diagnosis and treatment remains a continuous challenge to science researchers.

\section{Conflicts of Interest}

The authors declare that no conflicts of interest exist.

\section{Authors' Contributions}

Erbao Chen and Xiaojing $\mathrm{Xu}$ contributed equally to this work.

\section{References}

[1] L. A. Torre, F. Bray, R. L. Siegel, J. Ferlay, and J. Lortet-Tieulent, "Global cancer statistics, 2012," CA: A Cancer Journal for Clinicians, vol. 65, no. 2, pp. 87-108, 2015.

[2] N. Akamatsu, U. Cillo, A. Cucchetti et al., "Surgery and hepatocellular Carcinoma," Liver Cancer, vol. 6, no. 1, pp. 44-50, 2017.

[3] S. Schlesinger, K. Aleksandrova, T. Pischon et al., "Diabetes mellitus, insulin treatment, diabetes duration, and risk of 
biliary tract cancer and hepatocellular carcinoma in a European Cohort," Annals of Oncology, vol. 24, no. 9, pp. 2449-2455, 2013.

[4] C.-H. Chiang, L.-T. Lee, S.-H. Hung et al., "Opposite association between diabetes, dyslipidemia, and hepatocellular carcinoma mortality in the middle-aged and elderly," Hepatology, vol. 59, no. 6, pp. 2207-2215, 2014.

[5] M. M. Hassan, R. Abdel-Wahab, A. Kaseb et al., "Obesity Early in Adulthood Increases Risk but Does Not Affect Outcomes of Hepatocellular Carcinoma," Gastroenterology, vol. 149, no. 1, Article ID 59705, pp. 119-129, 2015.

[6] Y. Fang, Y. Feng, T. Wu et al., "Aflatoxin B1 Negatively Regulates Wnt/ $\beta$-Catenin Signaling Pathway through Activating miR33a," PLoS ONE, vol. 8, no. 8, p. e73004, 2013.

[7] K. Okuda, "Hepatocellular carcinoma," Journal of Hepatology, vol. 32, no. 1, pp. 225-237, 2000.

[8] S. Tanaka, K. Miyanishi, M. Kobune et al., "Increased hepatic oxidative DNA damage in patients with nonalcoholic steatohepatitis who develop hepatocellular carcinoma," Journal of Gastroenterology, vol. 48, no. 11, pp. 1249-1258, 2013.

[9] D. W.-H. Ho, R. C.-L. Lo, L.-K. Chan, and I. O.-L. Ng, "Molecular Pathogenesis of Hepatocellular Carcinoma," Liver Cancer, vol. 5, no. 4, pp. 290-302, 2016.

[10] D. Hanahan and R. A. Weinberg, "Hallmarks of cancer: the next generation," Cell, vol. 144, no. 5, pp. 646-674, 2011.

[11] H. Guo, N. T. Ingolia, J. S. Weissman, and D. P. Bartel, "Mammalian microRNAs predominantly act to decrease target mRNA levels," Nature, vol. 466, no. 7308, pp. 835-840, 2010.

[12] J. Krol, I. Loedige, and W. Filipowicz, "The widespread regulation of microRNA biogenesis, function and decay," Nature Reviews Genetics, vol. 11, no. 9, pp. 597-610, 2010.

[13] G. M. Borchert, W. Lanier, and B. L. Davidson, "RNA polymerase III transcribes human microRNAs," Nature Structural \& Molecular Biology, vol. 13, no. 12, pp. 1097-1101, 2006.

[14] M. Stewart, "Nuclear Export of Small RNAs," Science, vol. 326, no. 5957, pp. 1195-1196, 2009.

[15] J. Hou, L. Lin, W. Zhou et al., "Identification of miRNomes in human liver and hepatocellular carcinoma reveals miR-199a/b$3 \mathrm{p}$ as therapeutic target for hepatocellular carcinoma," Cancer Cell, vol. 19, no. 2, pp. 232-243, 2011.

[16] L. Wang, J. Yao, H. Sun et al., "miR-302b suppresses cell invasion and metastasis by directly targeting AKT2 in human hepatocellular carcinoma cells," Tumor Biology, vol. 37, no. 1, pp. 847-855, 2016.

[17] L. Wang, J. Yao, X. Shi et al., "MicroRNA-302b suppresses cell proliferation by targeting EGFR in human hepatocellular carcinoma SMMC-7721 cells," BMC Cancer, vol. 13, article no. 448, 2013.

[18] G.-G. Wu, W.-H. Li, W.-G. He et al., "Mir-184 post-transcriptionally regulates SOX7 expression and promotes cell proliferation in human hepatocellular carcinoma," PLoS ONE, vol. 9, no. 2, Article ID e88796, 2014.

[19] A. Petrelli, A. Perra, D. Cora et al., "MicroRNA/gene profiling unveils early molecular changes and nuclear factor erythroid related factor 2 (NRF2) activation in a rat model recapitulating human hepatocellular carcinoma (HCC)," Hepatology, vol. 59, no. 1, pp. 228-241, 2014.

[20] Y. Zhang, X. Guo, Z. Li et al., "A systematic investigation based on microRNA-mediated gene regulatory network reveals that dysregulation of microRNA-19a/Cyclin D1 axis confers an oncogenic potential and a worse prognosis in human hepatocellular carcinoma," RNA Biology, vol. 12, no. 6, pp. 643657, 2015.
[21] J. K. Kim, J. H. Noh, K. H. Jung et al., "Sirtuin7 oncogenic potential in human hepatocellular carcinoma and its regulation by the tumor suppressors MiR-125a-5p and MiR-125b," Hepatology, vol. 57, no. 3, pp. 1055-1067, 2013.

[22] D. Ngo-Yin Fan, F. Ho-Ching Tsang, A. Hoi-Kam Tam et al., "Histone lysine methyltransferase, suppressor of variegation 3-9 homolog 1, promotes hepatocellular carcinoma progression and is negatively regulated by microRNA-125b," Hepatology, vol. 57, no. 2, pp. 637-647, 2013.

[23] H. Yang, F. Fang, R. Chang, and L. Yang, "MicroRNA-140-5p suppresses tumor growth and metastasis by targeting transforming growth factor $\beta$ receptor 1 and fibroblast growth factor 9 in hepatocellular carcinoma," Hepatology, vol. 58, no. 1, pp. 205-217, 2013.

[24] M. Furuta, K. Kozaki, K. Tanimoto et al., "The tumor-suppressive miR-497-195 cluster targets multiple cell-cycle regulators in hepatocellular carcinoma," PLoS ONE, vol. 8, no. 3, Article ID e60155, 2013.

[25] H.-P. Li, X.-C. Zeng, B. Zhang et al., "miR-451 inhibits cell proliferation in human hepatocellular carcinoma through direct Suppression of IKK- $\beta$," Carcinogenesis, vol. 34, no. 11, pp. 24432451, 2013.

[26] R.-F. Liu, X. Xu, J. Huang et al., "Down-regulation of miR$517 \mathrm{a}$ and miR-517c promotes proliferation of hepatocellular carcinoma cells via targeting Pyk2," Cancer Letters, vol. 329, no. 2, pp. 164-173, 2013.

[27] R. Y. Liu, C. F. Diao, and Y. Zhang, "MiR-371-5p downregulates pre mRNA processing factor 4 homolog B (PRPF4B) and facilitates the G1/S transition in human hepatocellular carcinoma cells," Cancer Letters, vol. 335, no. 2, pp. 351-360, 2013.

[28] M. Rong, G. Chen, and Y. Dang, "Increased MiR-221 expression in hepatocellular carcinoma tissues and its role in enhancing cell growth and inhibiting apoptosis in vitro," BMC Cancer, vol. 13, no. 1, article 21, 2013.

[29] C. Giovannini, M. Minguzzi, M. Baglioni et al., "Suppression of p53 by Notch3 is mediated by Cyclin G1 and sustained by MDM2 and miR-221 axis in hepatocellular carcinoma," Oncotarget, vol. 5, no. 21, pp. 10607-10620, 2014.

[30] X.-W. Yang, G.-Z. Shen, L.-Q. Cao et al., "MicroRNA-1269 promotes proliferation in human hepatocellular carcinoma via downregulation of FOXO1," BMC Cancer, vol. 14, no. 1, article no. 909, 2014.

[31] K. Yuan, Z. Lian, B. Sun, M. M. Clayton, I. O. L. Ng, and M. A. Feitelson, "Role of mir-148a in hepatitis B associated hepatocellular carcinoma," PLoS ONE, vol. 7, no. 4, Article ID e35331, 2012.

[32] Y. Zhang, J. Dai, H. Deng et al., "miR-1228 promotes the proliferation and metastasis of hepatoma cells through a p53 forward feedback loop," British Journal of Cancer, vol. 112, no. 2, pp. 365374, 2015.

[33] W. Wang, L.-J. Zhao, Y.-X. Tan, H. Ren, and Z.-X. Qi, "MiR-138 induces cell cycle arrest by targeting cyclin D3 in hepatocellular carcinoma," Carcinogenesis, vol. 33, no. 5, pp. 1113-1120, 2012.

[34] L. Lin, H. Liang, Y. Wang et al., "MicroRNA-141 inhibits cell proliferation and invasion and promotes apoptosis by targeting hepatocyte nuclear factor- $3 \beta$ in hepatocellular carcinoma cells," BMC Cancer, vol. 14, no. 1, article no. 879, 2014.

[35] Y. Ma, X.-G. She, Y.-Z. Ming, and Q.-Q. Wan, "miR-24 promotes the proliferation and invasion of HCC cells by targeting SOX7," Tumor Biology, vol. 35, no. 11, pp. 10731-10736, 2014. 
[36] W. Zhang, K. Liu, S. Liu, B. Ji, Y. Wang, and Y. Liu, "MicroRNA133a functions as a tumor suppressor by targeting IGF-1R in hepatocellular carcinoma," Tumor Biology, vol. 36, no. 12, pp. 9779-9788, 2015.

[37] Y. Liu, Y. Hei, Q. Shu et al., "VCP/p97, down-regulated by microRNA-129-5p, could regulate the progression of hepatocellular carcinoma," PLoS ONE, vol. 7, no. 4, Article ID e35800, 2012.

[38] Y. M. Yang, C. G. Lee, J. H. Koo et al., “Galpha12 overexpressed in hepatocellular carcinoma reduces microRNA-122 expression via HNF4a inactivation, which causes c-Met induction," Oncotarget, vol. 6, no. 22, pp. 19055-19069, 2015.

[39] R. Nassirpour, P. P. Mehta, and M.-J. Yin, "miR-122 regulates tumorigenesis in hepatocellular carcinoma by targeting AKT3," PLoS ONE, vol. 8, no. 11, Article ID e79655, 2013.

[40] K. Ohta, H. Hoshino, J. Wang et al., "MicroRNA-93 activates cMet/PI3K/Akt pathway activity in hepatocellular carcinoma by directly inhibiting PTEN and CDKN1A," Oncotarget, vol. 6, no. 5, pp. 3211-3224, 2015.

[41] G. Shen, X. Rong, J. Zhao et al., "MicroRNA-105 suppresses cell proliferation and inhibits PI3K/AKT signaling in human hepatocellular carcinoma," Carcinogenesis, vol. 35, no. 12, pp. 27482755, 2014.

[42] J. Gong, J.-P. Zhang, B. Li et al., "MicroRNA-125b promotes apoptosis by regulating the expression of Mcl-1, Bcl-w and IL6R," Oncogene, vol. 32, no. 25, pp. 3071-3079, 2013.

[43] C. Cao, J. Sun, D. Zhang et al., "The long intergenic noncoding RNA UFC1, a target of microRNA 34a, interacts with the mRNA stabilizing protein $\mathrm{HuR}$ to increase levels of $\beta$-catenin in HCC cells," Gastroenterology, vol. 148, no. 2, pp. 415-426.e18, 2015.

[44] L. Chen, H. Guan, C. Gu, Y. Cao, J. Shao, and F. Wang, "miR-383 inhibits hepatocellular carcinoma cell proliferation via targeting APRIL," Tumor Biology, vol. 37, no. 2, pp. 2497-2507, 2016.

[45] L. Gramantieri, F. Fornari, M. Ferracin et al., "MicroRNA-221 targets Bmf in hepatocellular carcinoma and correlates with tumor multifocality," Clinical Cancer Research, vol. 15, no. 16, pp. 5073-5081, 2009.

[46] X. Xue, Y. Zhang, Q. Zhi et al., "MiR200-upregulated Vasohibin 2 promotes the malignant transformation of tumors by inducing epithelial-mesenchymal transition in hepatocellular carcinoma," Cell Communication and Signaling, vol. 12, no. 1, article no. 62, 2014.

[47] J. Liu, B. Ruan, N. You et al., "Downregulation of miR-200a induces EMT phenotypes and CSC-like signatures through targeting the $\beta$-catenin pathway in hepatic oval cells," PLoS ONE, vol. 8, no. 11, Article ID e79409, 2013.

[48] Y. Zhang, S. Takahashi, A. Tasaka, T. Yoshima, H. Ochi, and K. Chayama, "Involvement of microRNA-224 in cell proliferation, migration, invasion, and anti-apoptosis in hepatocellular carcinoma," Journal of Gastroenterology and Hepatology, vol. 28, no. 3, pp. 565-575, 2013.

[49] W.-C. Tsai, S.-D. Hsu, C.-S. Hsu et al., "MicroRNA-122 plays a critical role in liver homeostasis and hepatocarcinogenesis," The Journal of Clinical Investigation, vol. 122, no. 8, pp. 2884-2897, 2012.

[50] Y. Dang, D. Luo, M. Rong, and G. Chen, "Underexpression of miR-34a in Hepatocellular Carcinoma and Its Contribution towards Enhancement of Proliferating Inhibitory Effects of Agents Targeting c-MET," PLoS ONE, vol. 8, no. 4, Article ID e61054, 2013.

[51] J. Zhou, W. Li, J. Guo, G. Li, F. Chen, and J. Zhou, “Downregulation of miR-329 promotes cell invasion by regulating BRD4 and predicts poor prognosis in hepatocellular carcinoma," Tumor Biology, vol. 37, no. 3, pp. 3561-3569, 2016.

[52] F. Ni, H. Zhao, H. Cui et al., "MicroRNA-362-5p promotes tumor growth and metastasis by targeting CYLD in hepatocellular carcinoma," Cancer Letters, vol. 356, no. 2, pp. 809-818, 2015.

[53] H. Xia, L. L. P. J. Ooi, and K. M. Hui, "MicroRNA-216a/217induced epithelial-mesenchymal transition targets PTEN and SMAD7 to promote drug resistance and recurrence of liver cancer," Hepatology, vol. 58, no. 2, pp. 629-641, 2013.

[54] S. Tang, G. Tan, X. Jiang et al., "An artificial lncRNA targeting multiple miRNAs overcomes sorafenib resistance in hepatocellular carcinoma cells," Oncotarget , vol. 7, no. 45, pp. 7325773269, 2016.

[55] H. J. Bae, K. H. Jung, J. W. Eun et al., "MicroRNA-221 governs tumor suppressor HDAC6 to potentiate malignant progression of liver cancer," Journal of Hepatology, vol. 63, no. 2, pp. 408-419, 2015.

[56] L. Zhang, L. Yang, X. Liu et al., "MicroRNA-657 promotes tumorigenesis in hepatocellular carcinoma by targeting transducin-like enhancer protein 1 through nuclear factor kappa B pathways," Hepatology, vol. 57, no. 5, pp. 1919-1930, 2013.

[57] J. Ding, S. Huang, Y. Wang et al., "Genome-wide screening reveals that miR-195 targets the TNF- $\alpha / N F-\kappa B$ pathway by down-regulating $\mathrm{I} \kappa \mathrm{B}$ kinase alpha and TAB3 in hepatocellular carcinoma," Hepatology, vol. 58, no. 2, pp. 654-666, 2013.

[58] R.-M. Chang, H. Yang, F. Fang, J.-F. Xu, and L.-Y. Yang, "MicroRNA-331-3p promotes proliferation and metastasis of hepatocellular carcinoma by targeting $\mathrm{PH}$ domain and leucinerich repeat protein phosphatase," Hepatology, vol. 60, no. 4, pp. 1251-1263, 2014.

[59] Y. Fang, J.-L. Xue, Q. Shen, J. Chen, and L. Tian, "MicroRNA-7 inhibits tumor growth and metastasis by targeting the phosphoinositide 3-kinase/Akt pathway in hepatocellular carcinoma," Hepatology, vol. 55, no. 6, pp. 1852-1862, 2012.

[60] B.-F. Ning, J. Ding, J. Liu et al., "Hepatocyte nuclear factor $4 \alpha$-nuclear factor- $\kappa \mathrm{B}$ feedback circuit modulates liver cancer progression," Hepatology, vol. 60, no. 5, pp. 1607-1619, 2014.

[61] T.-C. Shih, Y.-J. Tien, C.-J. Wen et al., "MicroRNA-214 downregulation contributes to tumor angiogenesis by inducing secretion of the hepatoma-derived growth factor in human hepatoma," Journal of Hepatology, vol. 57, no. 3, pp. 584-591, 2012.

[62] G. Alpini, S. S. Glaser, J.-P. Zhang et al., "Regulation of placenta growth factor by microRNA-125b in hepatocellular cancer," Journal of Hepatology, vol. 55, no. 6, pp. 1339-1345, 2011.

[63] J.-H. Fang, H.-C. Zhou, C. Zeng et al., "MicroRNA-29b suppresses tumor angiogenesis, invasion, and metastasis by regulating matrix metalloproteinase 2 expression," Hepatology, vol. 54, no. 5, pp. 1729-1740, 2011.

[64] L. Bao, Y. Yan, C. Xu et al., "MicroRNA-21 suppresses PTEN and hSulf-1 expression and promotes hepatocellular carcinoma progression through AKT/ERK pathways," Cancer Letters, vol. 337, no. 2, pp. 226-236, 2013.

[65] Z.-T. Chai, J. Kong, X. Zhu et al., "MicroRNA-26a Inhibits Angiogenesis by Down-Regulating VEGFA through the PIK3C2 $\alpha /$ Akt/HIF-1 $\alpha$ Pathway in Hepatocellular Carcinoma," PLoS ONE, vol. 8, no. 10, Article ID e77957, 2013.

[66] R. Wang, N. Zhao, S. Li et al., "MicroRNA-195 suppresses angiogenesis and metastasis of hepatocellular carcinoma by inhibiting the expression of VEGF, VAV2, and CDC42," Hepatology, vol. 58, no. 2, pp. 642-653, 2013. 
[67] B. Zhou, R. Ma, W. Si et al., "MicroRNA-503 targets FGF2 and VEGFA and inhibits tumor angiogenesis and growth," Cancer Letters, vol. 333, no. 2, pp. 159-169, 2013.

[68] K. Zhu, Q. Pan, X. Zhang et al., "MiR-146a enhances angiogenic activity of endothelial cells in hepatocellular carcinoma by promoting PDGFRA expression," Carcinogenesis, vol. 34, no. 9, pp. 2071-2079, 2013.

[69] C. Du, Z. Lv, L. Cao et al., "MiR-126-3p suppresses tumor metastasis and angiogenesis of hepatocellular carcinoma by targeting LRP6 and PIK3R2," Journal of Translational Medicine, vol. 12, article 259, 2014.

[70] X. Yang, X. F. Zhang, and X. Lu, "MicroRNA-26a suppresses angiogenesis in human hepatocellular carcinoma by targeting hepatocyte growth factor-cMet pathway," Hepatology, vol. 59, no. 5, pp. 1874-1885, 2014.

[71] K. Zhu, Q. Pan, L.-Q. Jia et al., "MiR-302c inhibits tumor growth of hepatocellular carcinoma by suppressing the endothelialmesenchymal transition of endothelial cells," Scientific Reports, vol. 4, article 5524, 2014.

[72] J.-J. Yan, Y.-N. Zhang, J.-Z. Liao et al., "MiR-497 suppresses angiogenesis and metastasis of hepatocellular carcinoma by inhibiting VEGFA and AEG-1," Oncotarget, vol. 6, no. 30, pp. 29527-29542, 2015.

[73] L. Xu, S. Beckebaum, S. Iacob et al., "MicroRNA-101 inhibits human hepatocellular carcinoma progression through EZH2 downregulation and increased cytostatic drug sensitivity," Journal of Hepatology, vol. 60, no. 3, pp. 590-598, 2014.

[74] H. Han, D. Sun, W. Li et al., "A c-Myc-MicroRNA functional feedback loop affects hepatocarcinogenesis," Hepatology, vol. 57, no. 6, pp. 2378-2389, 2013.

[75] C. C.-L. Wong, C. Wong, E. K. Tung et al., "The microRNA miR139 suppresses metastasis and progression of hepatocellular carcinoma by down-regulating rho-kinase 2," Gastroenterology, vol. 140, no. 1, pp. 322-331, 2011.

[76] F. Fang, R.-M. Chang, L. Yu et al., "MicroRNA-188-5p suppresses tumor cell proliferation and metastasis by directly targeting FGF5 in hepatocellular carcinoma," Journal of Нераtology, vol. 63, no. 4, pp. 874-885, 2015.

[77] X. He, Y. Chang, F. Meng et al., "MicroRNA-375 targets AEG1 in hepatocellular carcinoma and suppresses liver cancer cell growth in vitro and in vivo," Oncogene, vol. 31, no. 28, pp. 33573369, 2012.

[78] A. M. Liu, Z. Xu, F. H. Shek et al., "MiR-122 targets pyruvate kinase M2 and affects metabolism of hepatocellular carcinoma," PLoS ONE, vol. 9, no. 1, Article ID e86872, 2014.

[79] Y. Mano, S. Aishima, N. Fujita et al., "Tumor-associated macrophage promotes tumor progression via STAT3 signaling in hepatocellular carcinoma," Pathobiology, vol. 80, no. 3, pp. 146154, 2013.

[80] S.-H. Lan, S.-Y. Wu, R. Zuchini et al., "Autophagy-preferential degradation of MIR224 participates in hepatocellular carcinoma tumorigenesis," Autophagy, vol. 10, no. 9, pp. 1687-1689, 2014.

[81] S.-H. Lan, S.-Y. Wu, R. Zuchini et al., "Autophagy suppresses tumorigenesis of hepatitis B virus-associated hepatocellular carcinoma through degradation of microRNA-224," Hepatology, vol. 59, no. 2, pp. 505-517, 2014.

[82] P. Yang, Q.-J. Li, Y. Feng et al., “TGF- $\beta$-miR-34a-CCL22 signaling-induced treg cell recruitment promotes venous metastases of HBV-positive hepatocellular carcinoma," Cancer Cell, vol. 22, no. 3, pp. 291-303, 2012.
[83] H. Yan, X. Dong, X. Zhong et al., "Inhibitions of epithelial to mesenchymal transition and cancer stem cells-like properties are involved in miR-148a-mediated anti-metastasis of hepatocellular carcinoma," Molecular Carcinogenesis, vol. 53, no. 12, pp. 960-969, 2014.

[84] M. Esteller, "Non-coding RNAs in human disease," Nature Reviews Genetics, vol. 12, no. 12, pp. 861-874, 2011.

[85] P. T.-Y. Law, A. K.-K. Ching, A. W.-H. Chan et al., "MiR145 modulates multiple components of the insulin-like growth factor pathway in hepatocellular carcinoma," Carcinogenesis, vol. 33, no. 6, pp. 1134-1141, 2012.

[86] L. Lim, A. Balakrishnan, N. Huskey et al., "MicroRNA-494 within an oncogenic microRNA megacluster regulates G1/S transition in liver tumorigenesis through suppression of mutated in colorectal cancer," Hepatology, vol. 59, no. 1, pp. 202215, 2014

[87] F.-Y. Huang, D. K.-H. Wong, W.-K. Seto, C.-L. Lai, and M.-F. Yuen, "Estradiol induces apoptosis via activation of miRNA23a and p53: Implication for gender difference in liver cancer development," Oncotarget, vol. 6, no. 33, pp. 34941-34952, 2015.

[88] L. Wu, Q. Wang, J. Yao, H. Jiang, C. Xiao, and F. Wu, "MicroRNA let-7g and let-7i inhibit hepatoma cell growth concurrently via downregulation of the anti-apoptoticprotein B-cell lymphomaextra large," Oncology Letters, vol. 9, no. 1, pp. 213-218, 2015.

[89] T. Cao, H. Li, Y. Hu, D. Ma, and X. Cai, "miR-144 suppresses the proliferation and metastasis of hepatocellular carcinoma by targeting E2F3," Tumor Biology, vol. 35, no. 11, pp. 10759-10764, 2014.

[90] R. Yuan, Q. Zhi, H. Zhao et al., "Upregulated expression of miR-106a by DNA hypomethylation plays an oncogenic role in hepatocellular carcinoma," Tumor Biology, vol. 36, no. 4, pp. 3093-3100, 2015.

[91] J.-N. Zhou, Q. Zeng, and H.-Y. Wang, "MicroRNA-125b attenuates epithelial-mesenchymal transitions and targets stem-like liver cancer cells through small mothers against decapentaplegic 2 and 4," Hepatology, vol. 62, no. 3, pp. 801-815, 2015.

[92] J.-Y. Huang, K. Zhang, D.-Q. Chen et al., "MicroRNA-451: Epithelial-mesenchymal transition inhibitor and prognostic biomarker of hepatocelluar carcinoma," Oncotarget, vol. 6, no. 21, pp. 18613-18630, 2015.

[93] T. Kogure, W. L. Lin, I. K. Yan, C. Braconi, and T. Patel, "Intercellular nanovesicle-mediated microRNA transfer: a mechanism of environmental modulation of hepatocellular cancer cell growth," Hepatology, vol. 54, no. 4, pp. 1237-1248, 2011. 


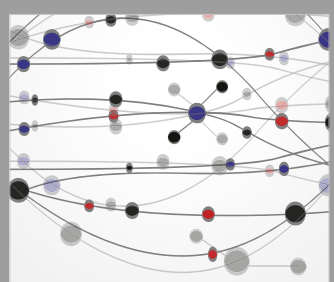

The Scientific World Journal
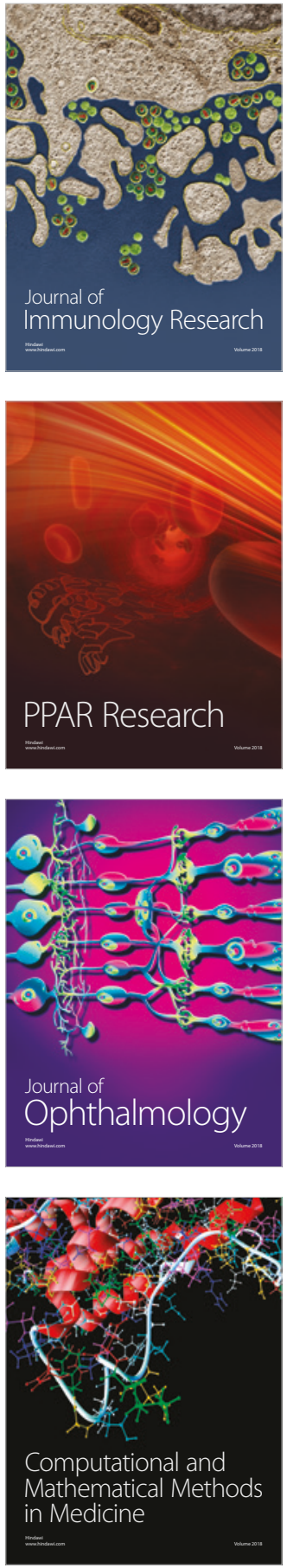

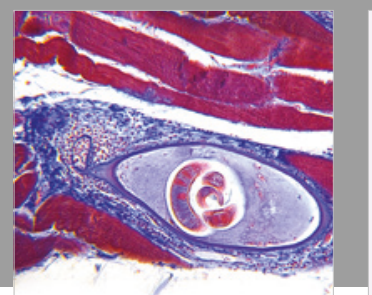

Gastroenterology Research and Practice

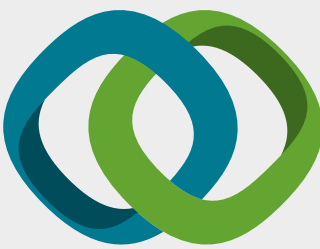

\section{Hindawi}

Submit your manuscripts at

www.hindawi.com
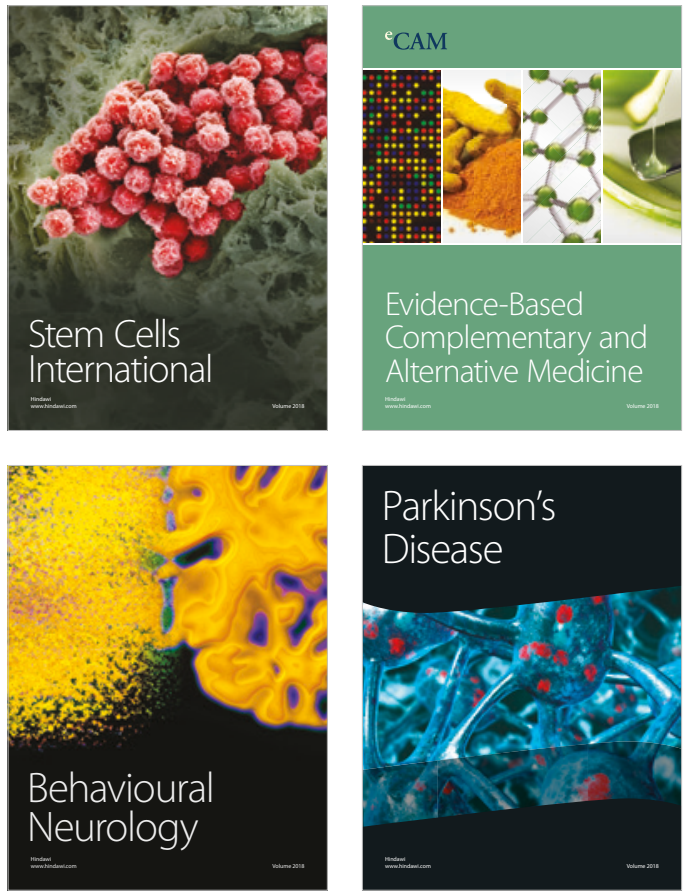

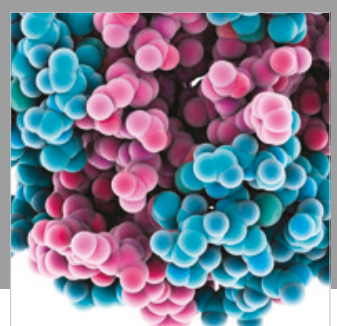

ournal of

Diabetes Research

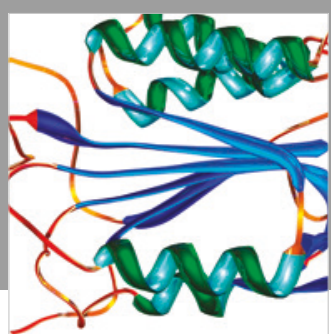

Disease Markers
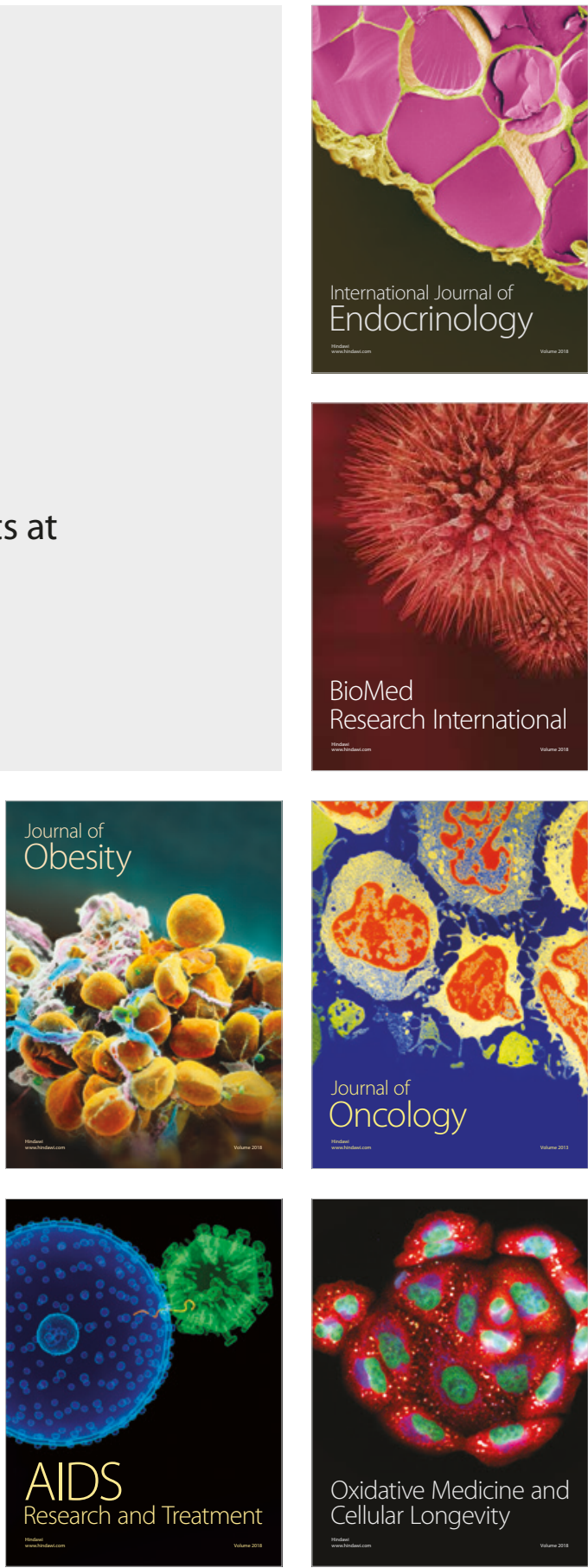